\title{
KINKS IN A PERIODICALLY MODULATED DISORDERED SYSTEM
}

\author{
Eva Majerníková ${ }^{\dagger}, \dagger \dagger$ and Jaroslav Riedel ${ }^{\dagger}$ \\ ${ }^{\dagger}$ Department of Theoretical Physics, Palacký University, Tř. 17. Listopadu 50, CZ-77207 Olomouc, Czech Republic \\ ${ }^{\dagger \dagger}$ Institute of Physics, Slovak Academy of Sciences, Dúbravská cesta 9, SK-84228 Bratislava, Slovak Republic \\ Boris A. Malomed \\ Department of Interdisciplinary Studies, Faculty of Engineering, Tel Aviv University, Tel Aviv 69978, Israel
}

(August 10, 2018)

\begin{abstract}
We consider a dc-driven damped sine-Gordon model with a small nonlinear spatial-disorder term, onto which a sinusoidal modulation is superimposed. It describes, e.g., a weakly disordered system with a regular grain structure. We demonstrate that, at the second order of the perturbation theory (with respect to the weak spatial disorder), the periodically modulated disorder gives rise to an effective periodic potential. Dynamics of a kink moving in this potential is studied in the overdamped limit, using the adiabatic approximation, the main objective being to consider depinning of a trapped kink. The analytical results are compared with direct dynamical simulations of the underlying model, as well as with numerical results using the collective-coordinate approach but without the mean-field approximation. It is found that a critical force for the depinning of a kink trapped by the periodically modulated weak spatial disorder is much larger than that predicted by the mean-field approximation.

PACS: 05.45.Y, 61.43.Bn, 71.55.Jv
\end{abstract}

\section{INTRODUCTION}

The interplay of nonlinearity and disorder is a wellknown topic in theoretical and experimental studies of solitonst 1 6. A paradigm one-dimensional model which has numerous physical applications and, in particular, allows one to consider interaction of topological solitons (kinks) with a random spatial inhomogeneity is a perturbed sine-Gordon (SG) equation. In the simplest case, the corresponding model takes a form 3 ,

$$
\Phi_{t t}-\Phi_{x x}+[1+\epsilon(x)] \sin \Phi=-(\Gamma / 8) \Phi_{t}-f,
$$

where $\Phi$ is a dynamical order parameter, $\Gamma$ is a dissipation constant, $f=$ const represents a driving force (external dc field), and the disorder function $\epsilon(x)$ is determined by its white-noise Gaussian correlations,

$$
\langle\epsilon(x)\rangle=0,\left\langle\epsilon(x) \epsilon\left(x^{\prime}\right)\right\rangle=\eta^{2} \delta\left(x-x^{\prime}\right),
$$

where $\eta$ is the disorder amplitude. In this work, the perturbation coefficients $\eta^{2}$ and $f$ will be treated as small parameters, while $\Gamma$ is not necessarily small. Note, that similar disordered models can be defined not only in the continuum form, but also on aperiodic lattices

In some systems, the spatial disorder can be subject to a superimposed regular (periodic) modulation. For instance, this may be the case if the underlying slightly disordered physical medium has a regular grain structure. Then, the model (11) is modified as follows, in the simplest case when the superimposed modulation is sinusoidal,

$$
\Phi_{t t}-\Phi_{x x}+[1+\epsilon(x) \sin (k x)] \sin \Phi=-(\Gamma / 8) \Phi_{t}-f,
$$

$2 \pi / k$ being the modulation period.

As concerns the form of the model (3), it is relevant to mention that, for any finite $k$, one can replace the modulation function $\sin (k x)$ by $\cos (k x)$, shifting $x \rightarrow$ $x+\pi / 2 k$. If the system is very long and $\epsilon(x)$ obeys the Gaussian correlations (2), the modulation functions are tantamount to each other, except the case $k=0$ (or $|k| \lesssim 1 / l$, if the system has a finite length $l$ ). Actually, for $k=0$ one comes back to the model (11) with the unmodulated spatial disorder.

Dynamics of topological nonlinear excitations in the form of kinks in the model (3) is a subject of the present work. In the lowest-order approximation, the random perturbation does not induce any effective potential for the kink. However, we demonstrate in section 2 that an effective periodic potential is generated at the second order of the perturbation theory. It is interesting that the amplitude of the effective potential vanishes at $k^{2}=1 / 2$.

In section 3 we study motion of the driven kink in this effective potential. The corresponding equation of motion is derived in the adiabatic approximation (treating the kink as a quasiparticle). This approximation is a version of the well-known collective-coordinate technique, which was applied to the (weakly disordered models in various contexts (see, e.g., Refs. only to derive an effective equation of motion, but also to study dynamical statistical characteristics of the kink We focus on the case when the overdamped approximation applies to the description of the kink motion (i.e., the inertia term in the corresponding equation of motion is neglected). In its direct form, the latter approximation assumes that the dissipation is stronger than the disorder, or, in terms of Eq. (11) $\Gamma \gg \eta$, in the generic case with $k \sim 1$. However, in this work we actually focus on a 
transition between the pinning and free-motion dynamical regimes (i.e., depinning of the trapped kink) with the increase of the driving force $f$. It is quite reasonable to assume that the inertia plays a negligible role in the depinning process, hence the overdamped approximation can be applied in a broad parametric region. Note that depinning of kinks trapped by the unmodulated spatial disofder in the model (1) was earlier studied in detail in Ref.B.

Section 4 displays results of numerical simulations of the model, which consists of two parts: direct dynamical simulations, and numerical analysis of the collectivecoordinate description without using the mean-field approximation. The latter approach yields accurate results for the critical (minimum) force $f_{\text {cr }}$ necessary to depin the trapped kink. The critical force turns out to be much larger than that predicted by the mean-field approximation. In particular, $f_{\text {cr }}$ is predicted by the mean-field approximation to scale $\sim \eta^{2}$ (see Eq. (2)), while the actually found critical force scales $\sim \eta$, the dependence on the modulation wave number $k$ (see Eq. (3)) also being quite different.

\section{SINE-GORDON KINK IN A PERIODICALLY MODULATED RANDOM POTENTIAL}

Equation (3) can be solved by iterations, assuming a small amplitude of the noise. The unperturbed kink solution is

$$
\Phi_{0}(x-\xi)=4 \tan ^{-1} \exp (x-\xi),
$$

$\xi$ being the coordinate of the kink's center.

A first-order correction to the kink waveform, $\phi_{1}=$ $\Phi(x)-\Phi_{0}$, satisfies the equation

$$
\left(\phi_{1}\right)_{t t}-\left(\phi_{1}\right)_{x x}+\cos \Phi_{0} \phi_{1}=-\epsilon(x) \sin (k x) \sin \Phi_{0} .
$$

To solve Eq. (5), we need its Green's function, which is defined in a standard way by means of the equation,

$$
\left\{\frac{\partial^{2}}{\partial t^{2}}-\frac{\partial^{2}}{\partial x^{2}}+V^{\prime \prime}\left[\Phi_{0}(x)\right]\right\} G\left(x, x^{\prime}, \tau\right)=\delta\left(x-x^{\prime}\right) \delta(\tau),
$$

where $\tau=t-t^{\prime}$ and $V(\Phi)=1-\cos \Phi$ is the SG potential. To simplify the formulas, we temporarily set $\xi=0$.

The necessary Green's function has been found in an explicit form by Flesch and Trullingert (see Eq. (3.29)): $G^{\mathrm{SG}}\left(x, x^{\prime}, \tau\right)$

$$
\begin{gathered}
=[\theta(\tau-|z|) / 2]\left\{J_{0}(s)+\beta_{2}\left[s J_{1}(s)-2 \tau \Lambda_{1}(w, s)\right]\right. \\
\left.-\beta_{3} \operatorname{sgn}(z)\left[-(\tau+|z|) J_{0}(s)+2 \tau \Lambda_{0}(w, s)\right]\right\} .
\end{gathered}
$$

Here, $z=x-x^{\prime}, s \equiv \sqrt{\tau^{2}-z^{2}}, w \equiv \tau-|z|, \theta(\tau)$ is the Heaviside's step function, and $\beta_{2} \equiv \tanh (x) \tanh \left(x^{\prime}\right)-$
1, $\beta_{3} \equiv \tanh \left(x^{\prime}\right)-\tanh (x)$. Further, $J_{0}(s)$ and $J_{1}(s)$ are the Bessel functions and $\Lambda_{0}(w, s)$ and $\Lambda_{1}(w, s)$ are the modified Lommel functions defined as

$$
\Lambda_{n}(w, s)=\sum_{m=0}^{\infty}\left(\frac{w}{s}\right)^{2 m+n} J_{2 m+n}(s) .
$$

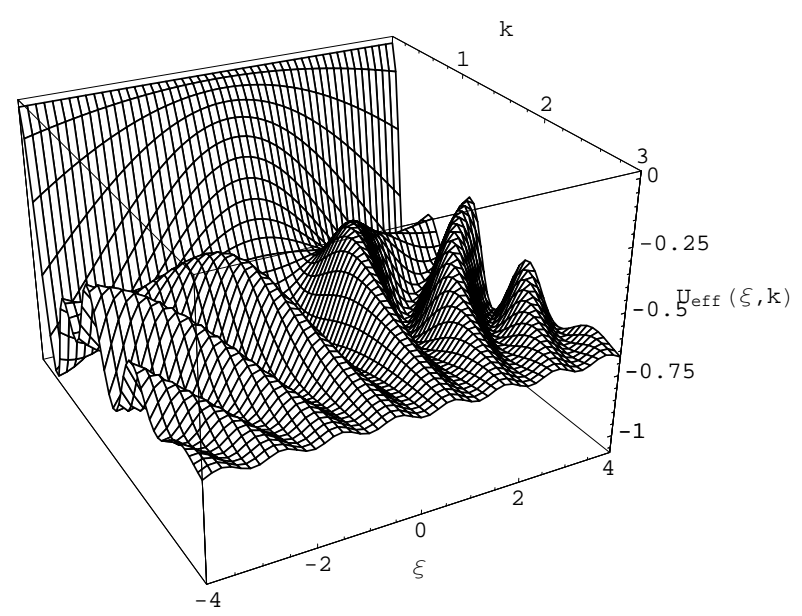

FIG. 1. The effective potential $U_{\text {eff }}(k, \xi)$.

Then, $\phi_{1}$ can be obtained in the form

$$
\begin{array}{r}
\phi_{1}(k, x)=-\int_{-\infty}^{\infty} d x^{\prime} \zeta\left(x^{\prime}\right) \sin \left(\phi_{0}\left(x^{\prime}-\xi\right)\right) \\
\times \sin \left(k x^{\prime}\right) G\left(x-x^{\prime}\right),
\end{array}
$$

where we have restored the kink's coordinate $\xi$.

The perturbation on the right-hand side of Eq.(5) gives rise, in a straightforward way 8 , to an effective potential for the kink treated as a quasiparticle:

$$
U_{\mathrm{eff}}(k, \xi)=\int_{-\infty}^{\infty} d x \epsilon(x) \sin (k x) \phi_{1}(k, x) \sin \Phi_{0}(x-\xi) .
$$

Then, inserting the expression (9) for $\phi_{1}(k, x)$ into Eq. (10), one obtains

$$
\begin{array}{r}
U_{\mathrm{eff}}(k, \xi)=-\int_{-\infty}^{\infty} d x \int_{-\infty}^{\infty} d x^{\prime} \epsilon(x) \epsilon\left(x^{\prime}\right) \sin \Phi_{0}(x-\xi) \\
\times \sin \Phi_{0}\left(x^{\prime}-\xi\right) \sin (k x) \sin \left(k x^{\prime}\right) G\left(x-x^{\prime}\right) .
\end{array}
$$

The potential (11) can be averaged with regard to (2). Using the elementary expressions for the SG kink, $\cos \Phi_{0}(x)=1-2 \operatorname{sech}^{2} x$ and $\sin \Phi_{0}=2 \sinh x \operatorname{sech}^{2} x$, yields the average potential 


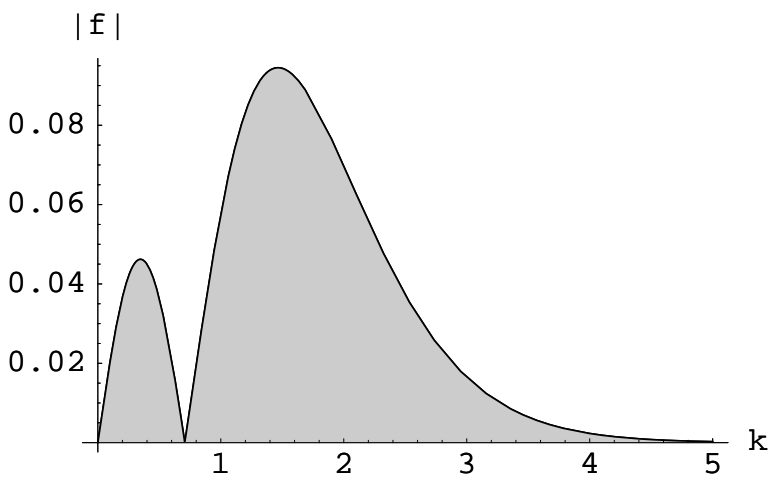

FIG. 2. The phase diagram for $\eta=1$. The regions of the free-motion $(|A / B|<1)$ and pinning $(|A / B|>1)$ regimes are, respectively, white and grey. At the boundary between them, $|A / B|=1$.

$$
\begin{aligned}
\left\langle U_{\text {eff }}(k, \xi)\right\rangle & =-2 \eta^{2} \int_{-\infty}^{\infty} d x \frac{\sinh ^{2}(x-\xi)}{\cosh ^{4}(x-\xi)} \sin ^{2}(k x) \\
& =-\frac{2 \eta^{2}}{3}\left[1-\pi k\left(1-2 k^{2}\right) \frac{\cos (2 k \xi)}{\sinh (\pi k)}\right] .
\end{aligned}
$$

Evidently, the average potential $\left\langle U_{\text {eff }}(k, \xi)\right\rangle(12)$ is periodic. It is plotted in Fig. 1 as a function of $\xi$ and $k$.

Minima of the potential (12) are at the points $\xi_{\min }=$ $(\pi / 2 k)(2 n+1), n=0,1, \ldots$, for $k^{2}<1 / 2$, and $\xi_{\min }=(\pi / k) n$ for $k^{2}>1 / 2$. Close to the minima, $\xi=\xi_{\min }+\delta, k \delta<<1$, the potential gives rise to small nearly harmonic oscillations with the frequency $\Omega^{2}=4 \eta^{2} \pi k^{2}\left|2 k^{2}-1\right| /(3 \sinh \pi k)$.

\section{DRIVEN MOTION OF THE KINK IN THE EFFECTIVE POTENTIAL: ADIABATIC APPROXIMATION}

In order to investigate uniformly driven damped motion of the kink in the effective periodically modulated random potential (12), we derive, in the framework of the standard adiabatic (quasi-particle) approximation $\mathrm{B}$, an equation of motion for the kink's center,

$$
8 \frac{d^{2} \xi}{d t^{2}}+\Gamma \frac{d \xi}{d t}=2 \pi f+\frac{4 \eta^{2} \pi k^{2}\left(1-2 k^{2}\right)}{3 \sinh (\pi k)} \sin (2 k \xi)
$$

(here, the "nonrelativistic" approximation, $(d \xi / d t)^{2} \ll 1$, is additionally adopted; nfte that the kink's mass in the present notation is $m=8 \mathrm{~B}$ ).

As it was explained above, we will chiefly be interested in the depinning threshold, when the inertia term in Eq. (13) may be neglected. Then, the equation of motion simplifies to

$$
\frac{d \xi}{d t}=\frac{2 \pi f}{\Gamma}+\frac{4 \eta^{2} \pi k^{2}\left(1-2 k^{2}\right)}{3 \Gamma \sinh (\pi k)} \sin (2 k \xi) \equiv B+A \sin (2 k \xi),
$$

where $A \equiv 4 \eta^{2} \pi k^{2}\left(1-2 k^{2}\right) /[3 \Gamma \sinh (\pi k)]$ and $B \equiv$ $2 \pi f / \Gamma$. Comparing Eqs. (13) and (14), one can verify that the inertia term is indeed negligible provided that $\Gamma \gg|A| k$. In the general case $(k \sim 1)$, this simply means $\Gamma \gg \eta$, i.e., the dissipative constant must be essentially larger than the amplitude of the spatial disorder.

A solution to Eq. (14) is obvious. One has to distinguish the cases (i) $|A|=|B|$, (ii) $|A|<|B|$, and (iii) $|A|>|B|$. The driving and potential terms dominate, respectively, in the regions (ii) and (iii), while (i) represents a border between them, characterized by the value

$$
f_{\mathrm{b}}=\left(2 k^{2} \eta^{2} / 3\right)\left|1-2 k^{2}\right| / \sinh (\pi k)
$$

of the driving field. The corresponding phase diagram is plotted, in terms of the underlying parameters $k$ and $f$, in Fig. 2, where the boundary between the regions (iii) (grey) and (ii) (white) corresponds to the critical field (15). Basic features of these regimes, that can be deduced in an analytical form from Eq. (14), are summarized below.
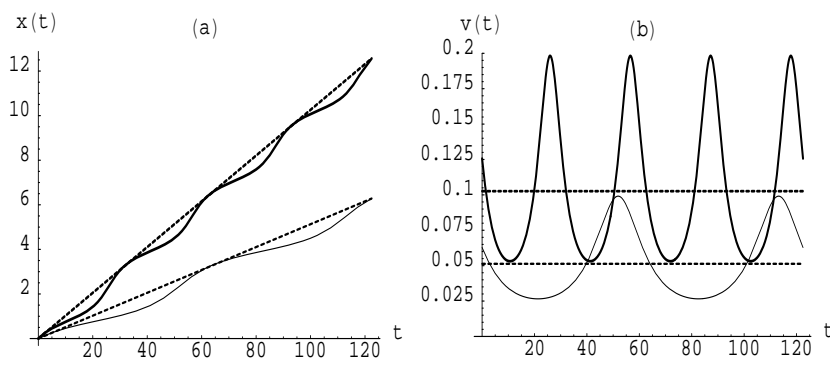

FIG. 3. Free (progressive) motion of the kink, shown in terms of $x(t)$ (a) and velocity $v(t)(\mathrm{b})$. Here and in the figures below the results are displayed for $\Gamma=10$ and $\Gamma=5$ by the thin and thick lines. The other parameters are $\eta=1, k=1$, and $f=0.1$.

(i) The border regime, $|A / B|=1$ : Eq. (14) gives rise to semi-stable fixed (stationary) points, $\xi_{\mathrm{s}}=(4 n-$ $1)(\pi / 4 k), n=0, \pm 1, \ldots$, which seem like attractors and repellers to the left and right of them, respectively. In this case, an analytical solution to Eq. (14) takes an implicit form,

$$
-\frac{\cos (2 k \xi)}{2 k A(1+\sin (2 k \xi))}=t-t_{0}
$$

$t_{0}$ being an initial moment.

(ii) The free (progressive) motion regime, $|A / B|<1$. In this case, Eq. (14) does not have stationary solutions. A time-dependent solution is $\tan (k \xi(t))$

$$
=-A / B+\sqrt{1-A^{2} / B^{2}} \tan \left[k \sqrt{B^{2}-A^{2}}\left(t-t_{0}\right)\right] .
$$

A typical example of this solution is displayed in Fig. 3. It represents a superposition of a linear trajectory and 


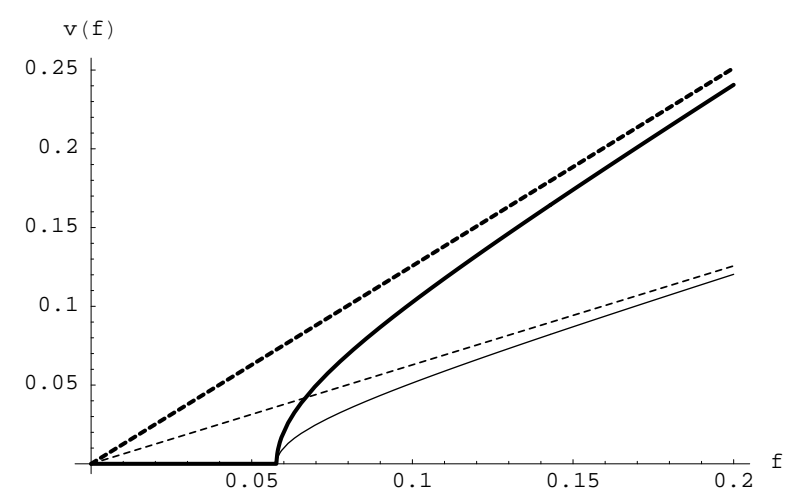

FIG. 4. The average velocity $\bar{v}$ vs. the driving field for $k=1, \eta=1$. The phase transition occurs at $f_{b}=0.0577264$.

periodic oscillations. The linear trajectory corresponds to the average velocity $\bar{v} \equiv \lim _{T \rightarrow \infty} \frac{1}{T}\left[\xi\left(t_{0}+T\right)-\xi\left(t_{0}\right)\right]=$ $\sqrt{B^{2}-A^{2}}$. In a neighborhood of the free-motion's border, at $f-f_{b} \rightarrow+0$, one has

$$
\bar{v} \approx(2 \pi / \Gamma) \sqrt{2 f_{\mathrm{b}}\left(f-f_{\mathrm{b}}\right)} .
$$

The average-velocity dependence as per Eq. (18) exhibits a typical mean-field critical (cusp) behavior close to the border, $\bar{v}$ playing the role of an order parameter (Figs. 4 and 5).

The time-periodic component of the velocity (see Fig. $3 \mathrm{~b})$ is characterized by the frequency $\omega=2 k \sqrt{B^{2}-A^{2}}$, whose critical behavior at $f-f_{b} \rightarrow+0$ is the same as that of the velocity (18). The mean-square deviation of the trajectory from the linear one, $\Delta \equiv \lim _{T \rightarrow \infty} \frac{1}{T} \int_{0}^{T} d t(\xi(t)-$ $\bar{v} t-\bar{\zeta})^{2}$, where $\bar{\zeta} \equiv \lim _{T \rightarrow \infty} \frac{1}{T} \int_{0}^{T} d t(\xi(t)-\bar{v} t)$, exhibits too the critical behavior as function of $f$. The $f$-dependencies of the reduced frequency $\omega / 2 \pi$ and of the deviation $\Delta$ are shown in Fig. 6.

Note that, representing the kink's law of motion in the form $\xi=\bar{v} t+\zeta(t)$, where $\zeta(t)$ is a time-periodic function with a small amplitude, one can expand the kink solution as follows (here, the "nonrelativistic" approximation $\bar{v}^{2} \ll 1$ is assumed):

$$
\begin{array}{r}
\phi(x, t)=4 \tan ^{-1}\{\exp [x-(\bar{v} t+\zeta(t))]\} \\
\approx 4 \tan ^{-1}\{\exp (x-\bar{v} t)\}+2 \zeta(t) \operatorname{sech}(x-\bar{v} t) .
\end{array}
$$

The small oscillating term in Eq. (19) may be a source for emission of radiation, provided that if contains spectral components with frequencies $\omega>18$. The Fourier decomposition of the source, defined as $\zeta(t)=$ $\sum_{n=-\infty}^{\infty} a_{n} \exp (i n \omega t)$, is illustrated by Fig. 7. Evidently, the density of the spectral components decreases with the distance from the boundary between the pinning and free-motion regions, i.e., with the increase of $f$. It is also obvious that the intensity of the generated radiation is extremely small, as the maximum frequency shown in

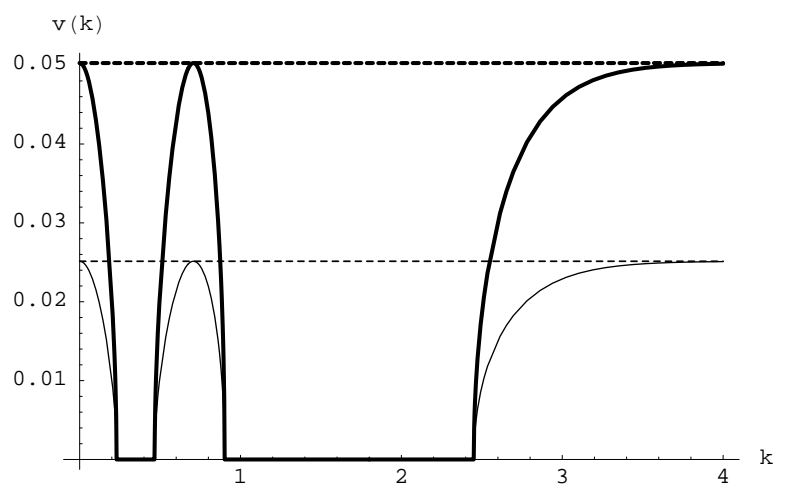

FIG. 5. The average velocity $\bar{v}$ vs. the modulation wave number $k$ for $f=0.04, \eta=1$.

Fig. 7 is $\approx 0.377$ of the threshold value, $\omega=1$, beyond which the emission of radiation takes place.

(iii) The pinning regime, $|A / B|>1$. In Fig. 5, one has the pinning $(\bar{v}=0)$ and free-motion $(\bar{v} \neq 0)$ regions of $k$ in accord with the diagram in Fig. 2. In the pinning regime, Eq. (14) exhibits two sets of fixed points: potential minima, $\xi_{\mathrm{a}}=(\pi / k) n-(1 / 2 k) \sin ^{-1}(B / A), n=$ $0, \pm 1, \ldots$, which are attractors (stable fixed points), and potential maxima, which are repellers (unstable points), $\xi_{\mathrm{r}}=(4 n-1) \pi / 4 k+(1 / 2 k) \sin ^{-1}(B / A)$. In the pinning regime, a time-dependent solution to Eq. (14) is (cf. Eq. (17))

$$
\begin{aligned}
& \tan (k \xi(t)) \\
& \quad=-A / B+\sqrt{A^{2} / B^{2}-1} \tan \left[k \sqrt{A^{2}-B^{2}}\left(t-t_{0}\right)\right] .
\end{aligned}
$$

This solution describes overdamped motion of a particle trapped in a well of the tilted potential $\left\langle U_{\text {eff }}\right\rangle$. The stationary state is reached after a short transient.

\section{NUMERICAL SIMULATIONS}

\section{A. Direct simulations of the perturbed sine-Gordon equation}

To get direct insight into the dynamics of the present weakly disordered model and check the above analytical results, we solved Eq. (3) numerically. For this purpose,
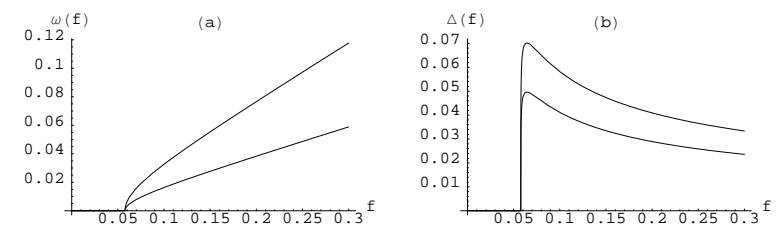

FIG. 6. Frequency of the oscillating part of the velocity and the mean square deviation of the trajectory from the linear one in the free-motion regime (ii), $\omega(f)$ (a) and $\Delta(f)$ (b) for $\eta=1, k=1$. 


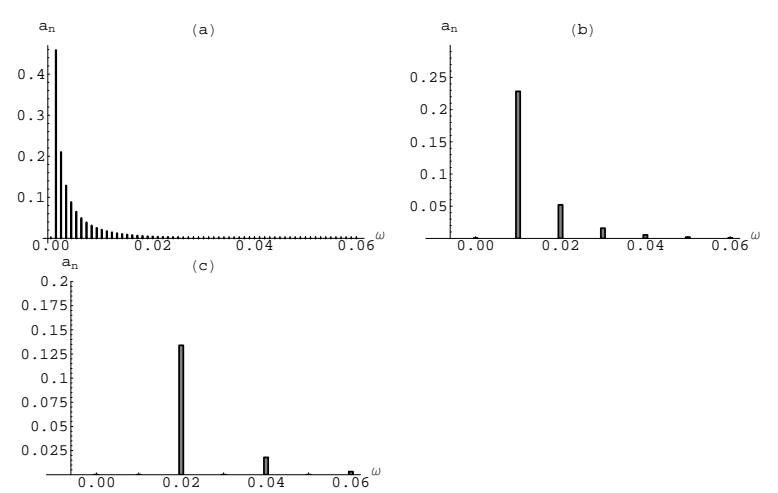

FIG. 7. Fourier spectra of the periodic part of the trajectory for $\eta=1, k=1$, and $\Gamma=10: f=0.057942$ (a), $f=0.076369(\mathrm{~b})$, and $f=0.115665$ (c).

Eq. (3) was transformed to a set of difference equations in the spatial coordinate (differential in the time coordinate) and solved by using the eighth-order explicit Runge-Kutta scheme10, with a stepsize control such that the time step was dynamically changed within the range from 0.05 to 0.3 . It has been chosen so that the relative (per one site) error at each step did not exceeded $10^{-10}$. In order to study the kink dynamics within rather big time intervals (for our computations the required time lap was $0 \leq t \leq 10^{4}$ ) we have used a shifting computational domain with boundaries which absorbed the outgoing waves.

At $t=0$, we took, for the initial conditions, the wellknown approximate (perturbative) static kink solution valid in the absence of the spatial disordert,

$$
\begin{aligned}
\Phi_{K}(s) & =\phi_{s}+4 \tan ^{-1}(\exp s), s=\frac{x-v_{0} t}{\sqrt{1-v_{0}^{2}}}, \\
v_{0}^{2} & =\left(1+(\Gamma / 2 \pi f)^{2}\right)^{-1},
\end{aligned}
$$

where

$$
\phi_{s} \equiv-\sin ^{-1} f
$$

takes into account the shift of the background under the action of the dc drive.

In the simulations we used 1000 different realizations of the disorder $\{\epsilon\}$ and then averaged the results over the realizations with equal weights.

In Fig. 8, we compare the kink velocity, as obtained from the direct simulations of Eq. (3), and those predicted by Eq. (14). The mean field velocity overestimates the exact one what is a typical mean field feature.

The numerically found time dependence of the kink's velocity in the localization regime is plotted in Figs. 9 and 10. The corresponding dependence of the inverse total distance travelled by the kink, in this regime, during the simulation time is shown, as a function of the driving force, in Fig. 11. It is evident from this figure that, while with small $f$ the kink quickly gets trapped, with the increasing $f$ it keeps moving for a much longer time.

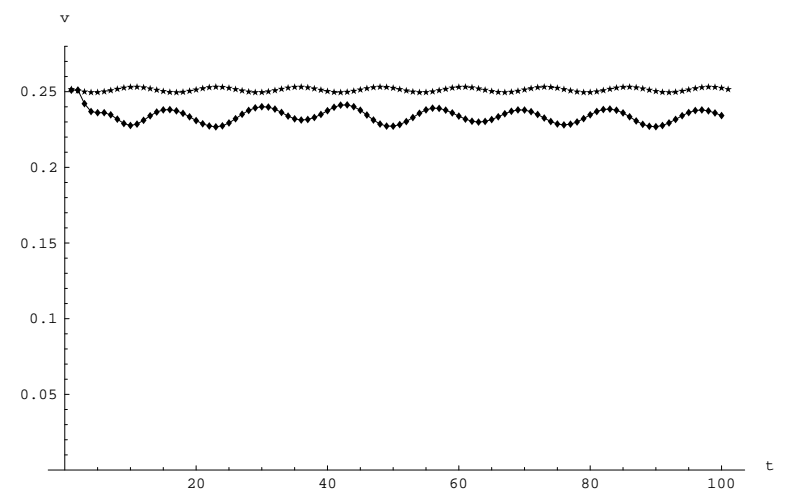

FIG. 8. Comparison of the velocity-vs.-time curves obtained from the numerically exact (diamonds) and adiabatic (stars) (Eq. (14)) solutions for $f=0.09, \eta=0.1, k=1$, and $\Gamma=2$.

When approaching the critical point, the trapping time enormously increases, therefore the direct numerical determination of the critical force $f_{\text {cr }}$ from the dynamical simulations is difficult. Besides, the calculations are constrained to a finite size of the system, hence the critical field would be size dependent. Moreover, at low $\Gamma$, when $f / \Gamma>1$, the one-kink model fails, as nucleation of kinkantikink pairs sets in with the accumulation of energy. Therefore, investigation of the critical region (transition between the trapped and freely moving kinks is not really possible on the basis of the direct simulations from Eq. (3).

\section{B. Numerical simulations of the collective coordinate equations}

Assuming low fields $f$, we will now follow the Rice's collective coordinate approach 12 , based on a modified ansatz for the kink, cf. Eqs. (4) and (21)

$$
\phi_{K}(x, t)=\phi_{s}+4 \tan ^{-1} \exp \left(\frac{x-\xi(t)}{L(t)}\right) .
$$

The difference from Eq. (4i) is that the present ansatz admits a variable kink's width $L(t)$. Inserting (23) into the model's Hamiltonian

$$
\begin{aligned}
H= & \int_{-\infty}^{\infty} d x\left\{\left(\Phi_{t}^{2}+\Phi_{x}^{2}\right) / 2+[1+\epsilon(x) \sin (k x)]\right. \\
& \left.\times\left(\cos \phi_{s}-\cos \Phi\right)-f x \Phi_{x}\right\}
\end{aligned}
$$

one arrives at the effective Hamiltoniand

$$
H_{\text {eff }}=\frac{L}{16} p_{\xi}^{2}+\frac{3 L}{4 \pi^{2}} p_{L}^{2}+U(L)+V(\{\epsilon\}, L, \xi)+2 \pi f \xi
$$

for the collective coordinates $\xi, L$ and the canonically conjugate momenta 


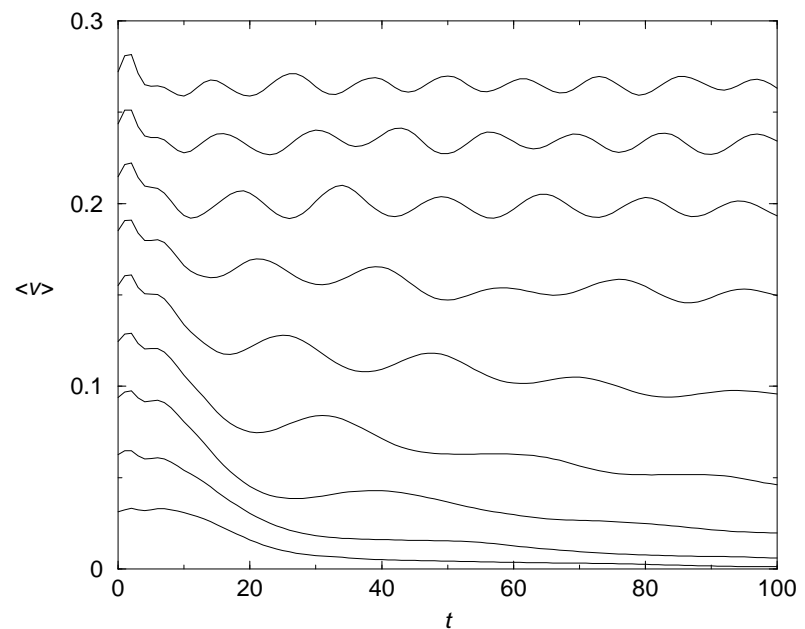

FIG. 9. Evolution of the soliton velocity for different values of the driving $f=0.1,0.2,0.3,0.4,0.5,0.6,0.7 . \Gamma=2, k=1$, $\eta=0.1$ and initial position $x_{0}=12.5$.

$$
p_{\xi}=\frac{8}{L} \frac{d \xi}{d t}, \quad p_{L}=\frac{2 \pi^{2}}{3 L} \frac{d L}{d t} .
$$

In the effective Hamiltonian (25),

$$
U(L)=4 L^{-1}+4 \cos \left(\phi_{s}\right) L
$$

is the effective potential in the case without disorder, and

$$
V(\{\epsilon\}, L, \xi)=\int_{-\infty}^{\infty} d x \epsilon(x) \sin (k x)\left(\cos \phi_{s}-\cos \Phi\right)
$$

is an effective random potential.

Taking into account the friction, we obtain the following system of equations of motion for the two kink's degrees of freedom:

$$
\begin{gathered}
\frac{d p_{\xi}}{d t}+\frac{\Gamma}{8} p_{\xi}+\frac{d}{d \xi} V(\{\epsilon\}, L, \xi)+2 \pi f=0, \\
\frac{d p_{L}}{d t}+\frac{\Gamma}{8} p_{L}+\frac{3 p 7_{L}^{2}}{4 \pi^{2}}+\frac{p_{\xi}^{2}}{16}+\frac{d}{d L}[U(L)+V(\{\epsilon\}, L, \xi)]=0 .
\end{gathered}
$$

Figs. 12 and 13 display comparison of the kink's velocities and widths, as obtained from the direct simulations of Eq. (3) and from simulations of the collective coordinate equations (29) and (30). A satisfactory agreement between the results of the two approaches is evident.

Eq.(29) makes it possible to determine the critical force $f_{\text {cr }}$ necessary to detrap the kink. For a single potential well (trap), $f_{\text {cr }}$ is simply equal to the maximum value of the local force induced by the trapping potential. However, a spatially random potential assumes the presence of a collection of the wells with randomly distributed parameters. Strictly speaking, one cannot uniquely define a global value of $f_{\text {cr }}$ in this case. Instead, it is necessary to consider a dynamical problem of gradually detrapping the kinks with the increase of the driving force 3 .

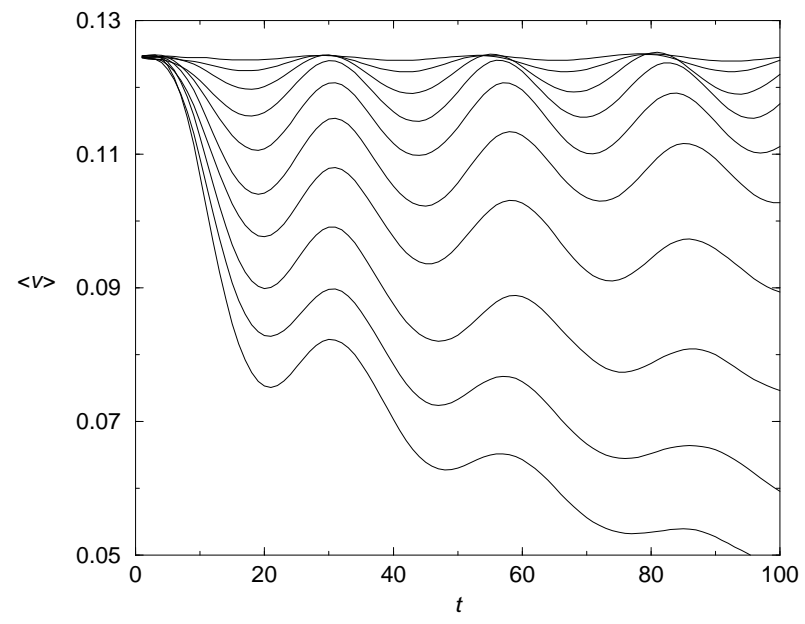

FIG. 10. The same as in Fig. 9, for $\eta=0.01, \ldots, 0.1$, $f=0.04, \Gamma=2, k=1$, and initial position $x_{0}=12.5$ for $5.10^{3}$ realizations of the random potential.

Nevertheless, it is possible to give a reasonable definition of the critical detrapping force as that which is equal to the local maximum value of the spatially random force, averaged over the length of the system (i.e., over all the local maxima):

$$
2 \pi f_{\mathrm{cr}}=\left\langle\frac{d}{d \xi} V(\{\epsilon\}, \xi)\right\rangle_{\max },
$$

the angular brackets standing for averaging. Here, the kink's width in the potential $V$ (28) should be set equal to the unperturbed value $L_{0}=1$.

If we suppose equal statistical weights for various local extrema of the function $d V / d \xi$ then the averaging theorem from Ref.11 (used e.g. in 6 ) can be applied directly, yielding

$$
\left\langle\frac{d}{d \xi} V(\{\epsilon\}, X)\right\rangle_{\max }=\sqrt{\frac{\pi}{2}} \frac{M_{2}}{\sqrt{M}_{4}},
$$

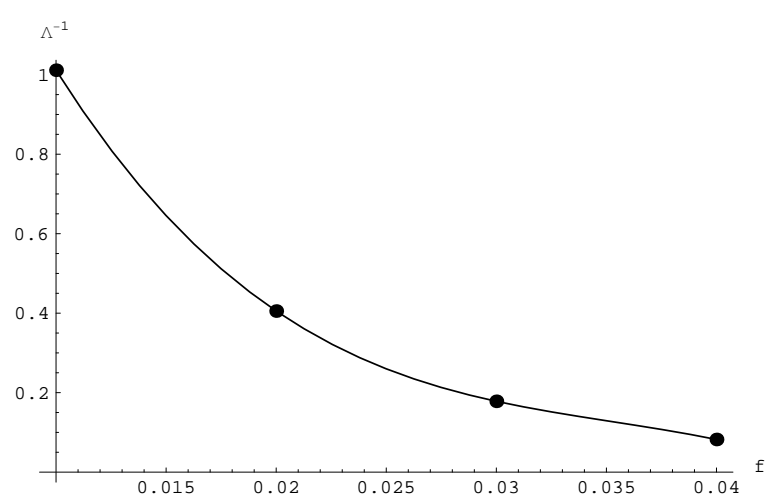

FIG. 11. Dependence of the inverse mean trajectory $\Lambda^{-1}$ on the force $f$ for $\Gamma=2, k=1, \eta=0.1$ and initial position $x_{0}=12.5$. 


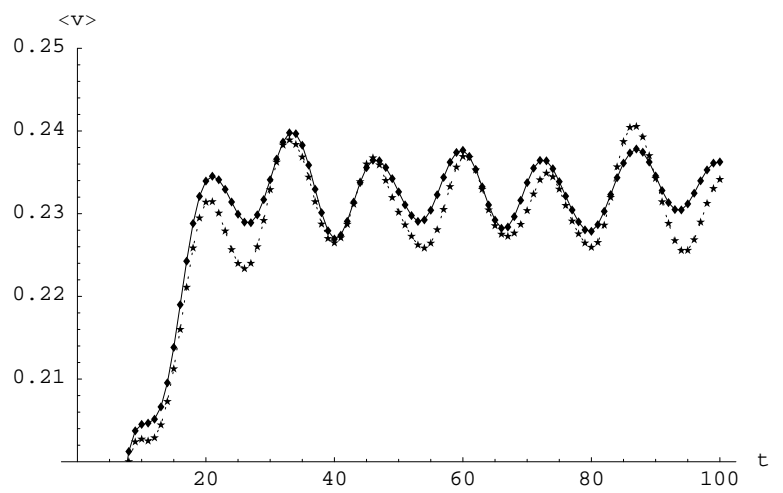

FIG. 12. Comparison of the averaged velocities vs. time from

the direct numerical simulations of Eq. (3) (squares) with that from the numerical solution of the collective coordinate equations (diamonds) for $\eta=0.1, f=0.08, k=1, \Gamma=2$.

where, in the present case,

$$
\begin{aligned}
& M_{2}=\left\langle\left(\frac{d^{2} V}{d \xi^{2}}\right)^{2}\right\rangle=4 \eta^{2} \int_{-\infty}^{\infty} d x \sin ^{2}(k x) q^{\prime \prime}(z), \\
& M_{4}=\left\langle\left(\frac{d^{3} V}{d \xi^{3}}\right)^{2}\right\rangle=4 \eta^{2} \int_{-\infty}^{\infty} d x \sin ^{2}(k x) q^{\prime \prime \prime}(z),
\end{aligned}
$$

and $2 q(z) \equiv\left(\cos \phi_{s}\right) \operatorname{sech}^{2}(z)-\left(\sin \phi_{s}\right) \sinh (z) \operatorname{sech}^{2}(z)$ (with $z \equiv(x-\xi) / L)$.

The integrals in (33) and (34) can be evaluated in a straightforward way,

$$
I_{j}=\left(1-f_{\mathrm{cr}}^{2}\right) Q_{j}+f_{\mathrm{cr}}^{2} R_{j}, \quad j=1,2 ;
$$

where

$$
Q_{1}=\frac{32}{21}-16 \cos (2 k \xi) S(\pi k L)\left(\frac{2}{3} P_{1}-\frac{2}{5} P_{2}+\frac{1}{35} P_{3}\right),
$$

$$
R_{1}=\frac{31}{21}-\cos (2 k \xi) S(\pi k L)\left(1-\frac{26}{3} P_{1}+\frac{32}{5} P_{2}-\frac{16}{35} P_{3}\right),
$$

$$
\begin{array}{r}
Q_{2}=\frac{256}{15}-128 \cos (2 k \xi) S(\pi k L)\left(\frac{2}{3} P_{1}-\frac{14}{15} P_{2}\right. \\
\left.+\frac{4}{21} P_{3}-\frac{2}{315} P_{4}\right), \\
R_{2}=\frac{254}{15}-\cos (2 k \xi) S(\pi K L) \\
\left(1-\frac{80}{3} P_{1}+\frac{896}{15} P_{2}\right. \\
\left.-\frac{256}{21} P_{3}+\frac{2.24^{2}}{9^{2} .35} P_{4}\right),
\end{array}
$$

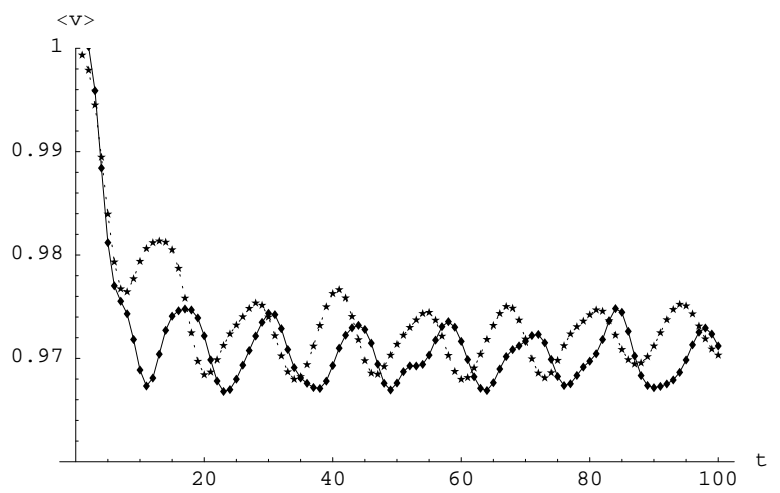

FIG. 13. The same for the averaged widths at the same values of parameters as in Fig. 13.

where $S(x)=x / \sinh x, P_{n}=\prod_{l=1}^{n}\left((k L)^{2}+l^{2}\right)$. Then, for $f_{\mathrm{cr}}$, one arrives at expression

$$
f_{c r}=\frac{\eta}{\sqrt{2 \pi L}} \frac{I_{1}\left(f_{\mathrm{cr}}^{2}\right)}{\sqrt{I_{2}\left(f_{\mathrm{cr}}^{2}\right)}} .
$$

The resulting function $f_{\mathrm{cr}}(k L, \xi)$ is plotted in Fig. 14 . To compare it with the mean-field result shown above in Fig. 2, we have set here $\xi=0$. A drastic difference between the two results is evident. Note also that the linear dependence of $f_{\text {cr }}$ on the disorder strength $\eta$ is in contrast to the quadratic dependence on $\eta$ in the mean field result (15).

It is relevant to stress that a characteristic critical depinning force, which was found for the model (1) with the unmodulated disorder in Ref.3, also scaled linearly with $\eta$. In other words, the depinning process is dominated by the underlying spatial disorder, rather than by the fact that the disorder is subject to a regular modulation. Thus, the mean-field approximation (alias, straightforward perturbation theory for the weak disorder) can not adequately describe depinning. Nevertheless, the modulation gives rise to a dependence of the pinning threshold upon the modulation period, see Fig. 14. In the limit of large $k$, the oscillations are dominated by the randomness and the case is reduced to that of the pure random noise. However, the most interesting is the region of the length scale competition $k^{-1} \sim L \leq 1$.

Note, that a strong nonperturbative response of solitons to weak random fluctuations in a narrow vicinity of a transition between different states is also known in other models, e.g., a dual-core nonlinear optical fibere.

\section{CONCLUSION}

In this work, we have introduced a dc-driven damped sine-Gordon model with a small nonlinear spatialdisorder term, on which a sinusoidal modulation is superimposed. It describes, e.g., a weakly disordered system with a regular grain structure. It was demonstrated that, 
in the mean-field approximation (second order of the perturbation theory), the periodically modulated disorder gives rise to an effective periodic potential. Dynamics of a kink moving in this potential was studied in the overdamped limit, using the adiabatic approximation, the main objective being to consider depinning of a trapped kink. The analytical results were compared against direct numerical simulations of the underlying model, as well as against numerical results using the collective-coordinate approach but without the mean-field approximation. It has been found that depinning of a kink trapped by the weak spatial disorder is, in reality, drastically different from what predicted by the mean-field approximation.

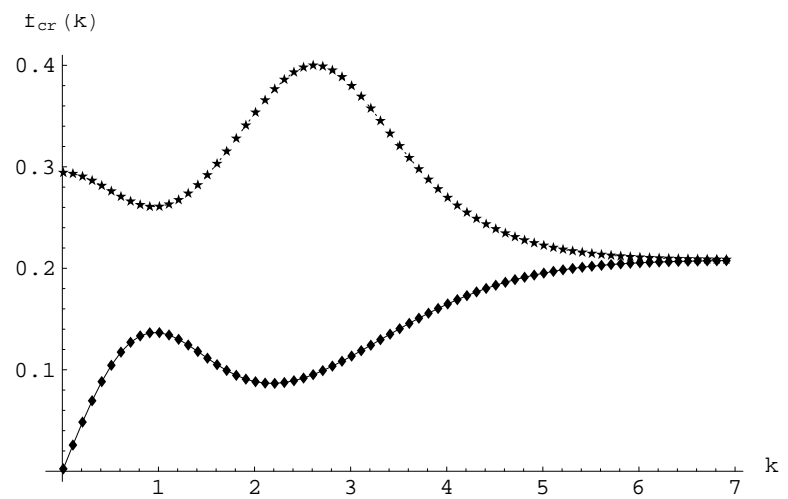

FIG. 14. Critical field $f_{\text {cr }}$ vs. wave number of the modulating field $k$. Full line is for $\xi=\pi / 2 k$, dashed for $\xi=0$. Quantitative as well as the qualitative difference to the mean field version in Fig. 2 is evident.

\section{ACKNOWLEDGEMENT}

We thank S.F. Mingaleev for discussions on numerical simulation methods. B.A.M. appreciates hospitality of the Department of Theoretical Physics at the Palacký University (Olomouc, The Czech Republic). The support by the grant No. 202/97/0166 of the Grant Agency of the Czech Republic and partially also by the grant No. 2/4109/98 of the VEGA Grant Agency is greatly acknowledged.

${ }^{1}$ Disorder and Nonlinearity (Springer Proceedings in Physics, vol. 39), ed. by A.R. Bishop, L.A. Krumhansl, and St. Pnevmatikos (Springer-Verlag.Berlin, 1989).

${ }^{2}$ V.V. Konotop and L. Vázquez. Nonlinear Random Waves (World Scientific.Singapore, 1994).

3 B.A. Malomed, Phys. Rev. B 39, 8011 (1989).

${ }^{4}$ S.A. Gredeskul, Y.S. Kivshar, L.M. Maslov, A. Sanchez and L. Vazquez, Phys. Rev. A 45, 8867 (1992).
${ }^{5}$ F. Dominguez-Adame, A. Sanchez, and Y.S. Kivshar, Phys.Rev. E 52, R2183 (1995).

${ }^{6}$ S.F. Mingaleev, Y.B. Gaididei, E. Majerníková and S. Shpyrko, Phys. Rev. B 59, 4074 (1999).

${ }^{7}$ R.J. Flesch and S.E. Trullinger, J. Math. Phys. 28, 1619 (1987).

${ }^{8}$ Y.S. Kivshar and B.A. Malomed, Rev. Mod. Phys. 61, 763 (1989).

9 A. Mostofi, B.A. Malomed, and P.L. Chu, Opt. Comm. 145, 274 (1998).

${ }^{10}$ J.R. Dormand and P.L. Prince, J. Comp. Appl. Math. 6, 19 (1980).

${ }^{11}$ O. Krée and C. Shoize, Mathematics of Random Phenomena (Reidel, Dordrecht, 1986).

12 M.J. Rice, Phys.Rev. B 28, 3587 (1983). 